\title{
LA POESÍA ESTRÓFICA DE IBN AL-'ARABI DE MURCIA
}

\author{
Por \\ FEDERICO CORRIENTE
}

\section{EL CEJEL XX\| (1)}

Uno de los muchos méritos del gran investigador que fue $S$. M. Stern, entre otros temas, de la poesía estrófica (hispano)-árabe, consistió en reparar en la presencia en el Diwān (2) del gran místico murciano, dentro de sus 29 poemas estróficos, de un único cejel (3), señalando asimismo su carácter de múârada o calco métrico del número 85 de Ibn Quzmān (4), cuyo preludio (matlala) le sirve de xarja. La noticia era importante, puesto que se venía afirmando que, así como Ibn al- ${ }^{C} \mathrm{Arab} i \mathrm{j}$ había introducido el uso "a lo divino" del muwašsah, habría correspondido a su algo más joven paisano aš-Sustari $\langle 5\rangle$ el mérito de hacer el primero lo propio con el cejel: como vemos, y aunque una golondrina no haga verano, habia tenido en esto al menos un

(1) Hemos decidıdo utilizar en adelante, como torma castellanizada del árabe /zajall, "cejel" y no "zejel», por dos razones: primera, evitar la imperfecta adaptación a la norma castellana que suponen la "z» y el acento grave, al que en vano se buscará consonante en nuestra lengua, frente a lo que ocurre si se acentúa como voz aguda, sobre todo cuando existe una segunda razón, para nosotros principal, y es que, aunque la norma oriental actual sea acentuar dicha palabra árabe en su primer silaba, sucedía lo contrario en andalusí ( $v$. nuestro A Grammatical Sketch of the Spanish-Arabic dialect bundle, 3.1.7.5.1.2 y 5.6 , donde se explica además el origen de su variante /záj// que, recogida por Alcalá, ed. Lagarde, p. 38 , ucantar zéjelu inspiró equivocadamente a quienes la castellanizaron tal cual, sin tener en cuenta su carácter secundario con respecto a la forma original /zajál/, única reflejada, v. gr., en el Vocabulista in Arabico, s. v. "cantilena" \& "versus"). Así también preferimos, por fidelidad a la pronunciación nativa, la acentuación "al-Andalús", como rechazamos las castelianizaciones "moaxaja" y ujarcha", por distorsionar intolerablemente la fonética árabe, sin tampoco ajustarse al sistema tradicional de transcripción casteliana de arabismos, por lo que nos parece menos arbitrario mantener las transcrípciones cientificas muwšsaha (para el género, muwašsaha, si se quiere, para la unidad) y xarja (o si se prefiere, barğga, jarŷa, etc., según el sistemá que se adoptel. En cuanto a la numeración XII con que identificamos este cejel, es ta correlativa dentro de los 29 poemas estróficos de Ibn al-ćArabī.

(2) Ed. Bülāq, $1271 \mathrm{~h}$. = 1855, pp. 214-5.

(3) Hispano-Arabic Strophic Poetry led. L. P. Harveyl, Oxford, 1974, pp. 85-6

(4) V. nuestra edición Gramática, métrica y texto del cancionero hispanoárabe de Aban Ouzmán (en adelante, GMT), Madrid, 1980, pp. 549-53, donde se escande como mustafcilun mustafcilun facolun, metro que, como veremos, es precisamente el utilizado por Ibn al- ${ }^{c}$ Arabĩ. La cita de Stern se refería, en un contexto más amplio, a otros casos de mucărada ea lo divino" de muwaš ahāt profanas famosas en la obra del mistico murciano. Lo mismo hizo aš-Šuštarī, destacando entre otros calcos rítmicos su número 96 , mucărada del número 24 de lbn Quzmăn.

(5) V. nuestro artículo «Observaciones sobre la métrica de aš-Suštarī» en Awrăq 5-6 (1982-31. 39-87. Por otrà parte, acabamos de dar a la imprenta una obra titulada la poesia estrófica (cejeles vio muwaš ahăt) atribuida al místico granadino as-Sustart, que contiene iexto, traducción y estudios de los materiales, hasta ahara accesibles sólo en la defectuosa edición de an-Našsár. 
momentáneo precursor si suponemos, como parece razonable, ya que Ibn al-cArabī murió en 638 h. (1240 d. C.), cuando aš-Suštari sólo tenía 30 años, que el poema del primero es algo anterior a los del segundo.

Pero Stern no dio una transcripción completa del poema, bastando para su propósito la de una sola estrofa, ni le habrla sido fácil ejecutarla con total éxito, como ya se advierte en la muestra, tanto por el carácter aún más titubeante en sus dias que hoy de la teoría métrica de estos géneros, como por el más imperfecto conocimiento que a la sazón se tenía del haz dialectal andalusí y, sobre todo, por las abundantes corrupciones que deturpan el texto de la edición del Diwắn de Ibn al-cArabí, al menos en estos pasajes. Sería altamente deseable una reedición y estudio de esta obra, que se beneficiaran tanto de los progresos hechos en el campo de la mística islámica, como de las actuales posibilidades de mejorar la presentación de los poemas estróficos que contiene, derivando de ellos la información pertinente sobre el desarrollo y características de estos géneros. En espera de que este progreso se produzca, nos parece posible y conveniente abordar un estudio preliminar de toda la poesía estrófica contenida en el DTwān de lobn al-'Arabì que, aun basándose en la edición citada y con sus limitaciones, puede resultar muy útil como investigación monográfica, y comparativa.

Procedemos, pues, en una primera entrega de dicho estudio, a dar nuestra transcripción, interpretación y anotación de este cejel, según la teoría y métodos propugnados en nuestra edición de ibn Quzmān, en nuestro artículo "The metres of the muwašah, an Andalusian adaptation of 'arüd" (6), y en los trabajos citados en la nota 5 .

\section{A) Texto (pp. 214-5 del Dïwăn):}

Wa qāla ayḍan min naịmi zzajali wahuwa laḥnu lcawămmi yaḍkuru fīhi alfāẓa ljawāhiri li'abī ḥāmid (7):

O ya tálib attahíq (8), aẓúr wujúdak, tará jamí annás 'abíd 'abidak.

1 qa`ádtu fi sáhil albáhr alaxợar, armát li amwáju addúrr alazhár, faqúltu: "la tagfál (9) yaqúti lașfár, wa'armi bíh (10), națlác (11) ila mahídak (11).

2 armát li falhín mácu dúrran akháb. faqúltu: uawíni ‘ánbarak alašháb». qálat: "nacám, ikkán ta`málli markáb min 'údak alfawwán, waxud̃, nazídak 1121

(6) En Journal of Atabic Literature 13 (1982), 76-72. Esta misma revista acaba de publicar otro articulo nuestro del mismo tema, titulado "Again on the metrical system of muwašsah and zajalm en 17 (1986) 34-49.

(7) Efectivamente, este cejel gira en torno a las alegorias creadas por aJ-Gazzali en su obra Kităb Jawăhir al-Qurcăn (trad. M. Abul Quasem, Londres, Kegan \& Paul, 1983, cap. (X), quien postula que el Corán contiene una amplia variedad de "perlasn y kgernasn: entre éstas, el alcrebite rojo, capaz de maravilosas transubstanciaciones, es el conocimiento de la esencia divina; el gran antidoto son aquellas alevas coránicas cuyos arqumentos sanan al hombre de ponzoñas y temores: el almizcle más fragante es la ciencia de la jurisprudencia, cuyo conocimiento proporciona fama e influencia que se extiende sin poder ocultarse y el áloe es el castigo divino que hace, a los que lo ven, procurar el Paraíso. De las otras alegorías, corindón o rubí, ámbar gris y crisólito, no cree necesario al-Gazżăli dar razón, dejando su interpretación al arbitrio del lector

(8) Ed. >thqu<, que no tolera el metro

(9) Ed. $>t^{\mathrm{c}} \mid<$, que hay que corregir.

$(10)$ Ed. $>$ fīh $<$, que hay que corregir.

(1) Ed. $>$ tț $10<$, que requiere enmienda.

(12) Alusión al principio islámico según el cual Dios gusta de quienes reciben su gracia y la agradecen \{v gr., Cor. $7 / 1441$, premiandoles con su incremento. 
3 zabárjadan axḍar, wamiskan (13) adfár (14), waddirriyáq (15) al'ákbar alláhu akbárc, fa'ana hu (16) Imațlúb. waqál wa`azzár: "lámm an (17) tirídni, qúli: ilík niridak.

4 wamši 'ala ssáhịi, wațlúb wa'aftás yaqúti alcaḥmár, lacálla tan`ás, facin laqáyt insán a'má wa'a'más. waqál: liman tațlúb? faqúl: lisídak."

5 ya țálib așșán'a, waffár (18) hayátak, wanz̧úr ila liksír 'alá șifátak, tijádu min dátak yđasrí lidátak, murábbac attarkíb 'ala wujúdak.

6 kibrítak al'aḥmár laqad [hu] (19) ma`lúm, wahúcala ttahqíq ajáli $\mathrm{ma}^{\mathrm{c}} \mathrm{dúm}$ : xafi zahár lal’áyn marmúz wamafhúm, fadába qad bánat juwár waridak (20), wađámmat asráru arkán jadídak.

7 al“ábd idá farráț la búdda yandám, wayámal alhíla, wala tifíd (21) tám. faqúltu: qál qáblak man qad taqaddám: «min áwil al'asúr anẓúr fi cidak; alhíla wáqt aḍḍíq ya (22) lís tifídak."

\section{B) Traducción:}

- Tú que procuras certeza (23), mira tu existencia y verás que todos son siervos de tus siervos.

1 Me senté a orillas del verde mar (24). $\checkmark$ sus olas me arrojaron relucientes perlas.

Yo dije: "No olvides mi topacio (25); arrójamelo, que suba a tu escape (26)m.

\footnotetext{
(13) Ed. >zbrjdk<, >msk<, que parece necesario corregir

(14) Curiosamente, y como indicio de lengua estandardizada pseudo-andalusí del cejel en muchos casos, no se refieja aqui la pronunciación dialectal lazfarl ( $v$. Sketch, núm. 57, donde por cierto se ha de corregir la cita de 10 como $100 / 2 / 1)$

(15) Ed. swdrryāc<, sin artículo que es necesario restituir. No recogen los diccionarios este alomorfo de /tiryãq/.

(16) Ed. >wa-<, que hay que enmendar.

(17) Ed. $>$ mn $<$

(18) Ed, >wbr<, que necesita enmienda.

(19) Adición métricamente necesaria y conveniente en el dialecto.

(20) Ed. >h. wär wzydk<, que ha de corregirse

(21) Ed. >yfyd<, que requiere enmienda.

(22) $\mathrm{Ed}$. $>$ mä<, mal entendido por copista oriental

(23) tahqia tecnicismo místico que implica la penetraçión de la verdad, al cesar la existencia separativa

(24) El mar, en esta alegoria, no es el océano de la Unidad u Omnipotencia, como a menudo en otros textos misticas, sino el Corán. que contiene todas estas perlas y gemas.

(25) Sic en el texto, vāqưt așfar, pero a la vista del original de al-Gazzāiĩ que se glosa, es probablemente una errata por yãqūt ahmar «corindón rojo o rubís (v, núm. 7$)$

(26) La rima ha requerido aquí la sustitución por mahíd del tecnicismo mistico budo "escape al mundo de lo sensible para alcanzar la penetración de la verdadn.
} 
2 Al punto me arrojaron además perlas grises, y dije: "Añádeme tu ámbar gris».

Dijo: "Sí, si me haces una nave (27)

de tu fragante áloe: toma y te daré además (28)

3 crisólito verde, almizcle, el más perfumado,

y la triaca mayor, que es [decir] "iDios es grande!

Pues yo soy el objetivo". Y dijo, reprendiendo:

"Cuando me busques, di: a $\mathrm{Ti}$ Te busco.

4 Ve por la orilla, busça registrando

mi rubí; tal vez te animes,

y si hallas a quien ciego y pitañoso (29)

te pregunte a quién buscas, di: a tu Señorn.

5 Tú que procuras ascesis, ahórrate la vida,

mira el elixir (30) en tus propiedades,

y verás que va de tu esencia a tu esencia,

con cuádruple composición, según tu existencia.

6 Tu alcrebite rojo (31) es conocido:

al cerciorarse, es el más excelso inexistente;

oculto es patente a la vista, en alegoría, pero comprendido

ahora junto a tu persona aparecen

$y$ se esparcen sus secretos en los cimientos de lo nuevo en ti.

7 Cuando el siervo se descuida, tiene que arrepentirse

y recurrir a ardides, cuando ya no sirven.

Yo digo: "Antes que tú ya hubo quien dijo:

Desde principios de āsür (32) prepara tu pascua,

que el ardid en el aprieto ya no te es útilm.

Métricamente, no parece dudosa la escansión de este cejel como mustafcilun mustafcilun facülun, una variedad de rajaz ya usada por lbn Quzmān (números 6 y 31, además del número 85), aunque curiosamente ausente en los poemas atribuidos a aš-Šštarī, al menos sin alguna modificación. Es llamativa la frecuencia con que en el segundo mustafilun aparece una "larga» teórica en la posición de breve, nada menos que 12 veces sobre 31 posibilidades, en $0 / 1$ lanz̧úr/, 1/1 /albáḥr/, 1/2 /addúrrl, 2/3/tacmálil, 4/1/aṭlúb/, 4/3 /acmál, 5/1/waffárl, 5/3/yasrí/, 6/3 /marmúzl, 6/5 larkán/, 7/3/man/ y $7 / 4$ /anzúrl. En aproximadamente la mitad de estos casos $11 / 1$, $1 / 2,4 / 3,5 / 1$ y $6 / 5)$ podría haberse dado la sustitución del pie mustafcilun por fắ-ilàtun: tales casos, que hemos detectado con frecuencia en los materiales atribuidos a aš-Sustrari, parecen haber constituido un recurso o licencia no rara en la composición de cejeles y muwašsahăt, con el que habrá que contar en el futuro al estudiar su métrica. Pero en todos los 12 casos se trata de sílabas normalmente átonas en andalusí, por lo que, según nuestra teoría, su presencia en posición de "breve» teórica era aceptable, si bien, no demasiado frecuente, por el hábito que tenían los autores cultos, a menudo simultaneadores de la composición en estos géneros y en poesía clásica, de respetar, siquiera ópticamente o mediante artificio (mugālața) (33), las

\footnotetext{
(27) La nave simbaliza aquil la disposición para adentrase en el mar del Corán, océano de la unidad

(28) V. núm. 12

(29) O sea, el hombre no iluminado por la iniciación mistica.

(30) Las metáforas inspiradas en la alquimia son frecuentes en el lenguaje alegórico de los místicos, lo que no implica un profundo conocimiento de aquella materia, pues en este poema parecen utilizarse como sinónimos /iksint y /kibrït ahmart.

(31) El «alcrebiten o «azufres urojon no parece ser, como afirma Dozy en su Supplément, el uorom, sino la piedra filosofal. mediante la cual se conseguían maravillosas transformaciones de sustancias.

(32) Sobre este término, como designación de los diez días del mes de Du-t-Hijja anteriores a la Fiesta del Sacrificio /Fíd a/adhàl. v. E. Garcia G., Todo Ben Quzmàn I, p. 429.

433) V. S al-Hilli, Kitäb al-cātill al-hăli wat-muraxxaş al-găli (Ed. W. Hoenerbach, Wiesbaden 1956), pp. 17-18.
} 
exigencias del carüạ. No deja de ser curioso que en este poema no se dé ningún caso parecido en el primer mustafilun, lo que podrian esgrimir los partidarios de la escansión cuantitativa estricta como prueba de que el metro real es :-_...-. .--, y asi suprimir aquella docena de pruebas a favor de la hipótesis del carü adaptado a una recitación acentual. Pero la misma lógica exigiría expliçar por qué no se aprovecha nunca tampoco la posibilidad de usar como ánceps la segunda sílaba de ambos mustafilun (34), ni la primera del primer mustafcilun (salvo tres casos), cuando la res. puesta a todas estas preguntas puede ser una dada ya hace tiempo (35), a saber, que la escasez de breves teóricas en dialecto, remediada mediante modificaciones de pies, mugâlața, o el recurso extremo de la "larga" átona en posición de breve, era muy significativa.

Aunque aún quedan muchas preguntas por responder para explicar a total satisfacción la naturaleza de la adaptación del carự a la poesía estrófica, seguimos pensando que nuestra hipótesis es la más económica, como se ve una vez más en este caso, puesto que explica el metro del poema dentro prácticamente de los esquemas conocidos y sin recurrir a una extrañísima combinación de pies, alguno de los cuales no puede ser xalïliano. Es también evidente que, salvo en el último pie del verso, había cierta tolerancia para las sustituciones de pies y otras anomalías en los segmentos previos, tolerancia tanto mayor cuanto menos clasicizante era el poema (muwašsah o cejel) (36), pero la misma perseverancia con que, en general, se respetan "cantidades" nos hace pensar que el ritmo no dependía exclusivamente del número de sílabas y del acento en el segmento final, como viene a ocurrir en la poesía castellana, sino que tenía una cierta presencia a lo largo de todo el verso: para los que creemos haber demostrado que el andalusí había sustituido la cantidad silábica por la tonicidad, es natural pensar que dicho ritmo dependiera de secuencias acentuales, en una transposición de las relaciones cuantitativas posicionales del carüớ xalitiano.

Desde el punto de vista estrófico, este cejel es también algo peculiar. En efecto, diríase que su estructura es aabbba, o sea, exactamente la propuesta por Stern rop. cit., p. 53), como propia del cejel (y que, efectivamente, tienen los más de (Q), pero, por otra parte, no sólo tiene xarja de estructura aa (contra lo normal, según Stern, op. cit., p. 54), donde asimismo se señalan las frecuentes excepciones en (Q), sino que además la estrofa 6 también tiene estructura bbbaa. Es difícil explicar esta anomalía, pues en las estrofas anteriores el sentido queda completo y no es fácil imaginar que todas havan perdido un segundo verso de vueltas; una explicación podría ser que la estrofa 7 haya sido sobreañadida a una primera versión más corta (de hecho, 5 estrofas parece haber sido la longitud más normal de estos poemas). En todo caso, si tenemos en cuenta la situación reftejada por ios materiales atribuidos a aš-Suštarī, en cuya poesía estrófica sé confunden cejel y muwašsah, tanto por la lengua (generalmente intermedia, no por hibridismo intencionado, sino por licencia métrica que permite el dialectalismo o clasicismo por conveniencias de metro y rima), como por la estructura estrófica (con claro predominio de aabbbaa y sus derivados) (37), parece razonable concluir que el cejel de Ibn al- ${ }^{\mathrm{C}} \mathrm{Arabi}$ representa una fase yio medio a mitad de camino entre lbn Quzmān y aš-Suštari, lo que encaja bien cronológicamente,

\footnotetext{
(34) No es buen ejemplo el /tijádui de 5/3, pues esta acentuación era a menudo reproducida con mater lectionis, si corría peligro el ritmo. Segun to que algunos han llamado išba

(35) En nuestro articulo "Acento y cantidad en la fonologia del hispano-árabe.... en Al-Andalus 41 (1976), 1-13.

1361 Esto se nota bien, por ejemplo, en la obra de aš-Šuštari que tiene, frente a poemas de caruọ casi irreprochable, otros como el 47. más populares e inspirados y con una aplicación más dialectạl de la adaptación del carúd.

$371 \mathrm{~V}$. los datos sobre este particular del artículo citado en nota 5
} 
sin perjuicio de que en épocas y medios posteriores cejel y muwašah pudieran aparecer nuevamente diferenciados (38).

En cambio, desde el punto de vista lingüístico, este poema se ajusta bastante estrictamente a la norma quzmāniana que condenaba el uso de la lengua clásica en el cejel (39), pudiendo afirmarse que todo su texto es dialectal andalusí (40). Entre los rasgos que lo confirman incuestionablemente, por no poder ser resultado de una lectura intencionadamente dialectalizante, tenemos:

a) Fonología: diptongo reducido de $4 / 4$ /sídak/ (v. Sketch $1.4 .4 / 5$ y 5.2.2., GMT 1.3.1.2.1); invariabilidad del artículo/al-/ en juntura en $2 / 1 /$ falhín/ (ed. "fälhịin «, $v$. Sketch 5.3.11.

b) Morfología: Sufijo /-ak/ en diversas rimas (v. Sketch 5.12.1).

c) Sintaxis: Construcciones de /lacálla/ en $4 / 2$ y /la búdda/ en $7 / 1$ sin sus correspondientes regímenes /v. Sketch 9.2.2. v 9.3.2 /laysa/; enfatizador /laqad/ en oración nominal ante /hu/ anafórico en $6 / 1$ (v. Sketch 7.4.4); /li-/ como marca de acusativo en $4 / 5$ ( $v$. Sketch 7.2.1 y GMT 3.1.3.2.2)

d) Léxico: romancismo/ya/ en $7 / 5$.

(38) Por ejemplo, éste parece ser el caso en los cejeles de Ibn ai-Xațíb contenidos en la Nufāọtat al-Jirāb lil itesis doctoral de Sa $a^{c}$ diyya Fagya en la Universidad Complutensel, al menos en cuanto a la lengua, pues su estructura de preludio y vueltas es idéntica.

(39) V. nuestra traducción, El Cancionero Hispanoárabe ide Ibn Quzmãn), Madrid, Editora Nacional, 1984, p. 42.

(40) No es excepción segura a esto $7 / 3$ /taqaddám/ por latqaddám/, que puede deberse a una ultracorrección de un copista oriental, a tratarse de una iectura original, ya que la lengua del cejel, especialmente la escrita fuera de al-Andalús, era a menudo un a imitación del andalusi, a veces bastante imperfecta. 